\title{
AN INEQUALITY IN THE COMPLEX DOMAIN
}

\section{CĂTĂLIN ŢIGĂERU}

\begin{abstract}
We prove the inequality $\left|1+z_{1}\right|+\left|1+z_{2}\right|+\left|1+z_{1} z_{2}\right| \geqslant\left|z_{1}\right|+\left|z_{2}\right|$, where $z_{1}, z_{2}$ are two arbitrary complex numbers. Consequently, it results that, if $z_{1}, \ldots, z_{n}$ are $n$ arbitrary com-
\end{abstract} plex number, then $\sum_{k=1}^{n}\left|1+z_{k}\right|+\frac{1}{n-1} \sum_{1 \leqslant i<j \leqslant n}\left|1+z_{i} z_{j}\right| \geqslant \sum_{k=1}^{n}\left|z_{k}\right|$.

Mathematics subject classification (2010): 26D15, 42B15.

Keywords and phrases: complex numbers; modulus.

\section{REFERENCES}

[1] D. S. Mitrinović, Analytic Inequalities, Springer-Verlag, 1970. 